\title{
Infrared Scene Projector Digital Model Mathematical Description
}

\author{
Mark A. Manzardo ${ }^{1}$, Brett Gossage ${ }^{1}$, J. Brent Spears $^{1}$, and Kenneth G. LeSueur ${ }^{2}$ \\ ${ }^{1} 555$ Sparkman Drive, Executive Plaza, Suite 1622 \\ Huntsville, AL 35816 \\ \{mmanzardo, bgossage, bspears\}@rttc.army.mil \\ ${ }^{2}$ US Army, Developmental Test Command \\ Redstone Technical Test Center \\ CSTE-DTC-RT-E-SA, Bldg. 4500 \\ Redstone Arsenal, AL 35898-8052 \\ klesueur@rttc.army.mil
}

\begin{abstract}
This paper describes the development of an Infrared Scene Projector Digital Model (IDM). The IDM is a product being developed for the Common High Performance Computing Software Support Initiative (CHSSI) program under the Integrated Modeling and Test Environments (IMT) Computational Technology Area (CTA). The primary purpose of the IDM is to provide a software model of an Infrared Scene Projector (IRSP). Initial utilization of the IDM will focus on developing non-uniformity correction algorithms, which will be implemented to correct the inherent non-uniformity of resistor array based IRSPs. Emphasis here is placed on how IRSP effects are modeled in the IDM.
\end{abstract}

\section{General Description of an Infrared Scene Projector (IRSP)}

In general, an IRSP can be broken down into the interaction between the following subsystems/components: a Software Control Subsystem (SCS), a Computer Image Generator Subsystem (CIGS), a Control Electronics Subsystem (CES), an Environmental Conditioning Subsystem (ECS), an Infrared Emission Subsystem (IRES), a Projection Optics Subsystem (POS), a Non-Uniformity Correction Subsystem (NUCS), a Mounting Platform Subsystem (MPS) and a System Under Test (SUT). Figure 1 provides an overview block diagram illustration of an IRSP. This figure also illustrates the typical interaction between the subsystems. Not all of these interactions are required; some are for ease of use, configuration management, system safety, or other specific needs. It is unlikely that any given IRSP utilizes all of the illustrated interfaces.

A more detailed description of each of these subsystems is provided in the simultaneously published companion paper: "Infrared Scene Projector Digital Model Development". 


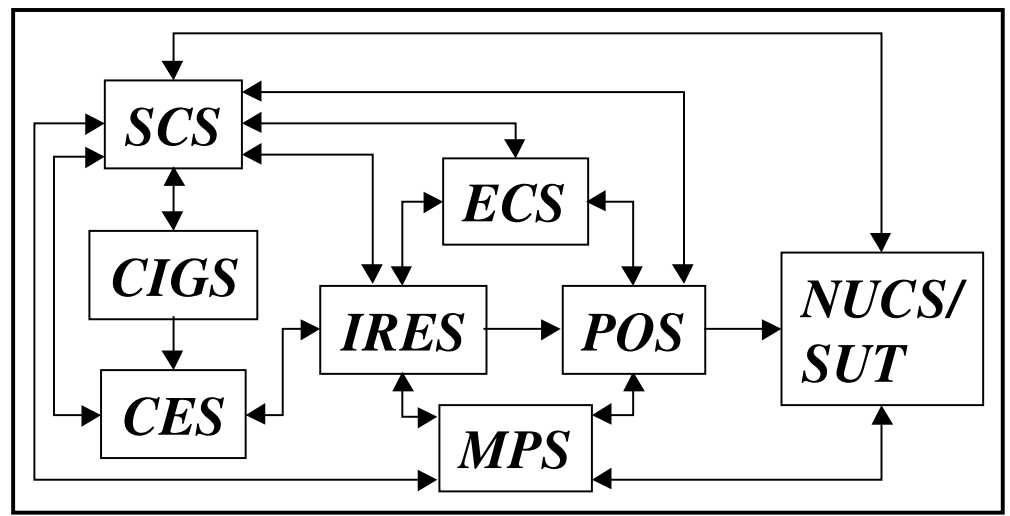

Fig. 1. Typical IRSP Block Diagram

\section{IRSP Digital Model (IDM) Mathematical Description}

The primary purpose of the IDM is to provide a digital model of the IRSP process allowing for NUC algorithm development while freeing up the actual hardware for utilization. The IDM will assume as input a temporal sequence of $2 \mathrm{D}$ digital image pixels with a 16-bit depth, the typical output of the CIGS. In other words, the IDM will not model the intricate process of actually generating the $2 \mathrm{D}$ rendered images. The IDM will provide as output a temporal sequence of 2D digital image pixels equivalent to the output of the NUCS camera or SUT. The bit depth of the output image will be dependent on the sensor. For example, the Agema 1000LR NUCS camera provides a 12-bit output digital image.

\subsection{IDM CIGS Model}

For IDM purposes, the CIGS is modeled simply as a sequence of two dimensional imagery. This is represented here as the image array DD02[, ].

\subsection{IDM CES Model}

For drive electronics systems that are used to exercise the Honeywell type resistor array, the 16-bit digital image signal is converted to analog drive voltages. This conversion is not perfect and therefore comes with a loss in resolution. For drive electronics systems that are used to exercise the Santa Barbara Infrared (SBIR) type resistor array, the 16-bit digital image signal is driven directly into the digital input of the resistor array. This effectively provides a lossless data path to the array. It should be noted that on the chip these digital signals are converted to analog signals to 
actually drive the resistor elements. This lossy effect has been described by SBIR as having the effect of adding equivalent noise to reduce the resolution of the system to 14.5 bits.

\subsubsection{Example Detailed CES Model Description of CSA Electronics}

Equation 1 provides a mathematical model describing the data path of DDO2 input 16-bit digital imagery through the drive electronics built by Computer Science and Applications (CSA). The output of this example CES model is the 2D array AIE[ , ], which has units of voltage.

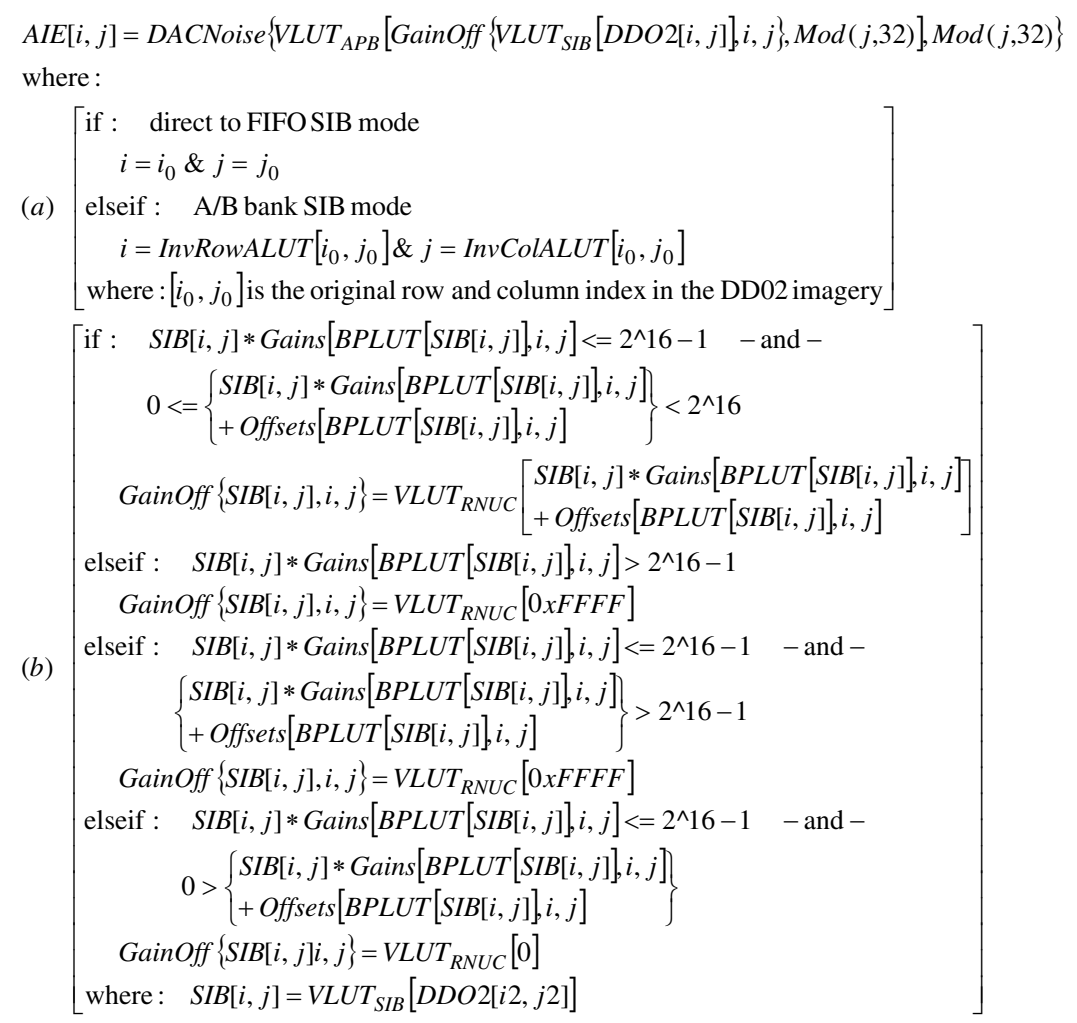

\subsection{IDM ECS Model}

As in a real IRSP, the IDM ECS acts primarily as a service unit. The ECS parameters (vacuum, IRES temperature, POS temperature, prevailing environment temperature ...) will be used to establish the necessary radiometric properties of the IRES, POS, and NUCS/SUT. The ECS set-points are considered to be temporally stationary (constant). However, due to the response and control loop(s) of real systems, there will be some drift and/or "noise" associated with the ECS parameters. To account for this the ECS parameters will be time varying values. 


\subsection{IDM MPS Model}

As in a real IRSP, the IDM MPS acts primarily as a service unit for alignment. The MPS parameters will be used to establish the relative spatial location of the IRES, POS, and NUCS/SUT. The MPS set-points are considered to be temporally stationary (constant). However, due to vibrations, there will be some drift and/or "noise" associated with the MPS parameters. To account for this the MPS parameters will also be time varying values.

\subsection{IDM IRES Model}

\subsubsection{IRES Bus-Bar Robbing Effect}

Bus-Bar Robbing is an effect common in the micro-resistor array technology. Basically, as the high current drive voltage bus is tapped into, by each FET along the bus, a voltage drop occurs dependent on how much current is drawn. This effect is a scene dependent effect and may require knowledge of the entire image before adequate compensation can be implemented. Although the Bus-Bar Robbing operation is not fully modeled yet, the IDM is configured to accommodate this effect by processing on the output of the CES (see Equation 1 above) as follows:

$$
\text { PostBusBar }[i, j]=\text { BusBarRob }\{\text { AIE }[]\}
$$

The empty brackets [ ] in this relationship indicate that the operator BusBarRob \{ \} may need the complete $A I E$ array (or some yet to be determined sub-array) to process the output for a given $[i, j]$ resistor. For a given $[i, j]$ resistor, the output of this operation is the discretely represented by array PostBusBar $[i, j]$.

\subsubsection{IRES Substrate Heating Effect}

IRES Substrate Heating is an effect which causes the local substrate to heat up when a large area of the array is driven at once. This is primarily due to the limitation of the heat sink to remove local heat. The local heat of a driven region will dissipate laterally across the heat sink to substrate boundary. This effect is a scene dependent effect. Although the IRES Substrate Heating operation is not fully modeled yet, the IDM is configured to accommodate this effect by processing on the output of the CES after the Bus-Bar Robbing effect as follows:

$$
\operatorname{PreNU}[i, j]=\text { SubHeat }\{\text { PostBusBar }[]\}
$$

The empty brackets [ ] in this relationship indicate that the operator SubHeat \{ \} may need the complete PostBusBar array (or some yet to be determined sub-array) to process the output for a given $[i, j]$ resistor. For a given $[i, j]$ resistor, the output of this operation is the discretely represented by array $\operatorname{PreNU}[i, j]$. 
NOTE: It may be alternatively sufficient to add an additional system blur to account for this effect. Adding addition blur effects are discussed later.

\subsubsection{IRES Non-uniformity Effect}

The primary issue surrounding the utilization of an IRSP is the inherent output NonUniformity (NU) that exists between the resistors when driven to the same input. This NU is a complex unknown input/output relationship, which differs for each resistor. For IDM implementation purposes, each resistor is assigned a set of NU coefficients of a yet to be determined functional form. The modeled output after NU is represented by:

$$
\operatorname{PostNU}[i, j]=\operatorname{ArrayN} U\{\operatorname{PreNU}[i, j], N U \operatorname{Coefs}[i, j, 1 \ldots N]\}
$$

The functional form used will be determined by empirical measurements. For a given [i, j] resistor, the output of this operation, ArrayNU\{ \}, is the discretely represented array PostNU[i, $j]$.

\subsubsection{IRES Fill Factor Effect}

Each resistor does not emit energy as a point source. The energy comes from the distributed area of the resistive material. To represent this effect, Post $N U[i, j]$ is represented as a two dimensional sum of dirac delta functions that is convolved with the analytically continuous FillFactor function. NOTE: The exact form of the FillFactor function will differ between different array designs. Up until now, the IRSP image representation has been in a unitless discretely sampled coordinate system. It is advantageous at this point to represent the energy coming off the array in the angular coordinate system in collimated space after the POS. The description of signal after incorporating the Fill Factor effect becomes:

$$
\begin{aligned}
\operatorname{PostArray}(\alpha, \beta)= & \left(\sum_{i=0}^{N_{i}-1 N_{j}-1} \operatorname{PostNU}[i, j] \delta\left(\alpha-\frac{i-\delta i_{\text {Array }}}{\text { iScale }_{\text {Array }}}, \beta-\frac{j-\delta j_{\text {Array }}}{j \text { Scale }_{\text {Array }}}\right)\right) * * \\
& \text { FillFactor }\left(\frac{\alpha}{\text { SScale }_{\text {Array }}}, \frac{\beta}{\beta \text { Scale }_{\text {Array }}}\right)
\end{aligned}
$$

It is important to note that at this point the IRSP signal is represented as an analytically continuous function. There is no theoretical limit to the spatial resolution used to represent this as a discretely sampled image for viewing purposes. However, any attempt to represent this discretely is subject to sampling and aliasing artifacts. To mitigate or limit such artifacts appropriate reconstruction filtering techniques should be used. As it will be shown later, these artifacts are not issues for the IDM's intended use. 


\subsection{IDM POS Model}

\subsubsection{POS Transmission and Geometric Aberrations Effects}

The POS will impose transmission, geometric aberrations, distortion, and diffraction effects. The diffraction effect is not implemented because diffraction will occur as a function of the POS and SUT optics combined. Since, an ideal POS will overfill or match the entrance pupil of an SUT, diffraction will occur at the SUT and is accounted for there. Similarly, the distortion effect is cumulative between the POS and SUT and is accounted for later at the system level. The POS may have a transmission that varies as a function of field of view. It is accounted for here as a multiplicative two dimensional function $\tau_{P O S}()$. The geometric aberrations effect is accounted for by convolution with another two dimensional analytical function $\operatorname{GPSF}_{P O S}()$. The description of the IRSP signal coming out of the POS becomes:

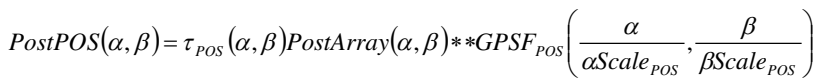

$$
\begin{aligned}
& \operatorname{PostPOS}(\alpha, \beta)=\tau_{\text {POS }}(\alpha, \beta)\left(\sum_{i=0}^{N_{i}-1 N_{j}-1} \operatorname{PostNU}[i, j] \delta\left(\alpha-\frac{i-\delta i_{\text {Array }}}{\text { SScale }_{\text {Array }}}, \beta-\frac{j-\delta j_{\text {Array }}}{j \text { Scale }_{\text {Array }}}\right)\right) * * \\
& \left(\begin{array}{l}
\text { FillFactor }\left(\frac{\alpha}{\text { dScale }_{\text {Array }}}, \frac{\beta}{\beta \text { Scale }_{\text {Aray }}}\right) * * \\
\operatorname{GPSF}_{P O S}\left(\frac{\alpha}{\alpha \text { Scale }_{\text {POS }}}, \frac{\beta}{\beta \text { Scale }_{P O S}}\right)
\end{array}\right)
\end{aligned}
$$

\subsection{IDM SUT Model}

\subsubsection{SUT Optics Transmission, Geometric Aberrations, and Diffraction Effects}

Similar to the POS, the SUT optics will have transmission, geometric aberration, distortion, and diffraction effects. As discussed earlier distortion effects will be handled later at the system level. Similar to the POS, transmission is accounted for here as a multiplicative two dimensional function $\tau_{S U T}()$. The combined SUT geometric aberrations and diffraction effects are accounted for here by convolution with another two dimensional analytical function $P S F_{S U T}()$. The description of the IRSP signal after SUT optics becomes: 


$$
\begin{aligned}
& \operatorname{PostSUTOptics}(\alpha, \beta)=\tau_{\text {System }}(\alpha, \beta) \operatorname{PostPOS}(\alpha, \beta) * * \operatorname{PSF}_{\text {SUT }}\left(\frac{\alpha}{\text { aScale }_{S U T}}, \frac{\beta}{\beta \text { Scale }_{S U T}}\right) \\
& \operatorname{PostSUTOptics}(\alpha, \beta)=\tau_{\text {System }_{\text {S }}}(\alpha, \beta)\left(\sum_{i=0}^{N_{i}-1 N_{j}-1} \operatorname{PostNU}[i, j] \delta\left(\alpha-\frac{i-\delta i_{\text {Array }}}{\text { SScale }_{\text {Array }}}, \beta-\frac{j-\delta j_{\text {Array }}}{j \text { Scale }_{\text {Array }}}\right)\right) * * \\
& \left(\begin{array}{l}
\text { FillFactor }\left(\frac{\alpha}{\alpha \text { Scale }_{\text {Array }}}, \frac{\beta}{\beta \text { Scale }_{\text {Array }}}\right) * * \\
\operatorname{GPSF}_{P O S}\left(\frac{\alpha}{\alpha \text { Scale }_{\text {POS }}}, \frac{\beta}{\beta \text { Scale }_{\text {POS }}}\right) * * \\
\operatorname{PSF}_{U U T}\left(\frac{\alpha}{\alpha \text { Scale }_{S U T}}, \frac{\beta}{\beta \text { Scale }_{S U T}}\right)
\end{array}\right)
\end{aligned}
$$

where:

$$
\tau_{\text {System }}(\alpha, \beta)=\tau_{\text {SUT }}\left(\alpha-\Delta \alpha_{\text {POS } 2 S U T}, \beta-\Delta \beta_{\text {POS2SUT }}\right) \tau_{P O S}(\alpha, \beta)
$$

NOTE: $\Delta \alpha_{\text {POSZSUT }}$ and $\Delta \beta_{\text {POSZSUT }}$ represent some potential registration shift between the Projection Optics and the SUT Optics. Assuming the SUT output image is centered on the SUT Optics, this will be the same equivalent shift of the detectors described later.

\subsubsection{SUT Detector Active Area Effect}

Similar to the resistor array fill factor effect, the detector does not detect at a point, but rather over some active area. The efficiency of this active area may not be continuous. It is therefore, represented here as a general two dimensional convolution function. The description of the IRSP signal just after detection becomes:

$$
\begin{aligned}
& \text { PostSUTDetector }(\alpha, \beta)=\text { PostSUTOptics }(\alpha, \beta) * * \text { DetectArea }_{\text {SUT }}\left(\frac{\alpha}{\alpha_{\text {Scale }} \text { SUT }}, \frac{\beta}{\beta \text { Scale }_{\text {SUT }}}\right) \\
& \operatorname{PostSUTDetector}(\alpha, \beta)=\tau_{S_{\text {ystem }}}(\alpha, \beta)\left(\sum_{i=0}^{N_{i}-1 N_{j}-1} \operatorname{PostNU}[i, j] \delta\left(\alpha-\frac{i-\delta i_{\text {Array }}}{i \text { Scale }_{\text {Array }}}, \beta-\frac{j-\delta j_{\text {Array }}}{j \text { Scale }_{\text {Array }}}\right)\right) * *
\end{aligned}
$$

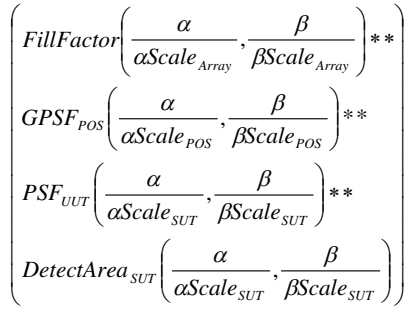

$$
\begin{aligned}
& \operatorname{PostSUTDetector}(\alpha, \beta)=\tau_{S_{\text {System }}}(\alpha, \beta)\left(\sum_{i=0}^{N_{i}-1 N_{j}-1} \sum_{j=0} \operatorname{PostNU}[i, j] \delta\left(\alpha-\frac{i-\delta i_{\text {Array }}}{\text { SCale }_{\text {Array }}}, \beta-\frac{j-\delta j_{\text {Array }}}{j \text { Scale }_{\text {Array }}}\right)\right) * * \\
& \text { System }(\alpha, \beta) \\
& \operatorname{PostSUTDetector}(\alpha, \beta)=\tau_{S_{\text {Strtem }}}(\alpha, \beta)\left(\sum_{i=0}^{N_{i}-1 N_{j}-1} \operatorname{PostNU}[i, j] \operatorname{System}\left(\alpha-\frac{i-\delta i_{\text {Array }}}{i \text { Scale }_{\text {Array }}}, \beta-\frac{j-\delta j_{\text {Array }}}{j \text { Scale }_{\text {Array }}}\right)\right)
\end{aligned}
$$

where: 


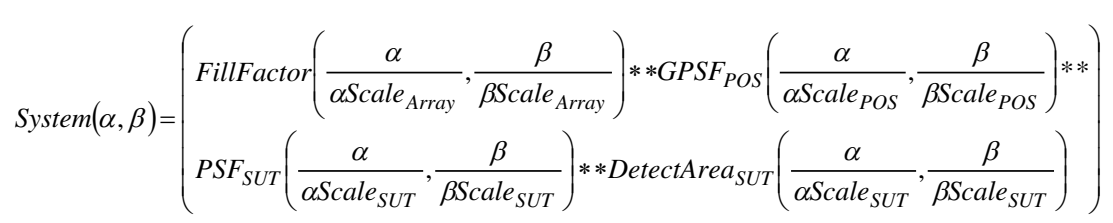

NOTE: Additional System Blur effects can easily be added to the System function as necessary via additional two dimensional convolution functions.

\subsubsection{SUT Discrete Sampling Effect}

Representing the image as discretely sampled by the SUT at the $[k, l]$ detector is accommodated by multiplication with an appropriately shifted dirac delta function. The description of the signal as discretely sampled by the SUT becomes:

$$
\begin{aligned}
& \left.\operatorname{PostSUTSamp}_{k}, l\right]=\delta\left(\alpha-\frac{k-\delta k_{S U T}}{k \text { Scale }_{S U T}}, \beta-\frac{l-\delta l_{S U T}}{\text { lScale }_{S U T}}\right) \operatorname{PostSUTDetector}(\alpha, \beta)
\end{aligned}
$$

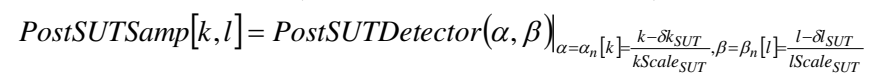

$$
\begin{aligned}
& \text { PostSUTSamp }[k, l]=\tau_{\text {System }}\left(\alpha_{n}[k], \beta_{n}[l]\right) \times \\
& \left(\sum_{i=0}^{N_{i}-1 N_{j}-1} \operatorname{PostNU}[i, j] \operatorname{System}\left(\alpha_{n}[k]-\frac{i-\delta i_{\text {Array }}}{\text { SScale }_{\text {Array }}}, \beta_{n}[l]-\frac{j-\delta j_{\text {Array }}}{j \text { Scale }_{\text {Array }}}\right)\right)
\end{aligned}
$$

At this point the signal is once again represented as a discretely sampled image that can be readily calculated and viewed. However, it is advantageous to first account for full system distortion effects. In addition the above form requires a double summation extending over the entire resistor array for each $[k, l]$ detector. A simplification to this form is presented below as well.

\subsubsection{SUT Noise Effect}

The SUT will add noise to the output signal. In the IDM implementation this operation, SUTNoise \{ \}, remains to be developed. However, as a place holder the IDM will process the image as follows:

$$
\text { PostSUTNoise }[k, l]=\text { SUTNoise }\{\text { PostSUTSamp }[]\}
$$

\subsubsection{SUT Digitization Effect}

Most SUTs of interest will also digitize the image in some form or fashion, even if this means external digitization of an RS170 image. In the IDM implementation this operation, SUTDig \{ \}, remains to be developed. However, as a place holder the IDM will process the image as follows:

$$
\text { Output }[k, l]=\operatorname{SUTDig}\{\text { PostSUTNoise }[]\}
$$




\subsection{Full System Distortion Effect}

The above representation is for an IRSP without any distortion, which is impractical. To incorporate overall system distortion effects, the nominal sampling location for the $[k, l]$ detector of $\left(\alpha=\alpha_{n}[k], \beta=\beta_{n}[l]\right)$ can be replaced by the actual distortion effected sampling location of $\left(\alpha=\alpha_{d}[k, l], \beta=\beta_{d}[k, l]\right)$. The following gives an example of how to incorporate overall IRSP distortion effect:

$$
\begin{aligned}
& \alpha_{d}[k, l]=C_{0}+C_{1} \alpha_{n}[k]+C_{2} \beta_{n}[l]+C_{3} r_{n}[k, l]+C_{4} \alpha_{n}^{2}[k]+C_{5} \beta_{n}^{2}[l]+ \\
& C_{6} \alpha_{n}[k] \beta_{n}[l]+C_{7} \alpha_{n}[k] r_{n}[k, l]+C_{8} \beta_{n}[l] r_{n}[k, l]+C_{9} r_{n}^{2}[k, l], \\
& \beta_{d}[k, l]=D_{0}+D_{1} \alpha_{n}[k]+D_{2} \beta_{n}[l]+D_{3} r_{n}[k, l]+D_{4} \alpha_{n}^{2}[k]+D_{5} \beta_{n}^{2}[l]+ \\
& \text { where: } \\
& D_{6} \alpha_{n}[k] \beta_{n}[l]+C_{7} \alpha_{n}\left[k r_{n}[k, l]+C_{8} \beta_{n}[l] r_{n}[k, l]+D_{9} r_{n}^{2}[k, l]\right. \\
& r_{n}[k, l]=\sqrt{\alpha_{n}^{2}[k]+\beta_{n}^{2}[l]}, \alpha_{n}[k]=\frac{k-\delta k_{S U T}}{k \text { Scale }_{S U T}}, \beta_{n}[l]=\frac{l-\delta l_{S U T}}{\text { lScale }_{S U T}}
\end{aligned}
$$

The $\mathrm{C}$ and $\mathrm{D}$ coefficients can be measured or modeled. With the incorporation of the distortion effect, the signal as discretely sampled by the SUT is:

$$
\begin{aligned}
& \text { PostSUTSamp }[k, l]=\text { PostSUTDetector }\left.(\alpha, \beta)\right|_{\alpha=\alpha_{d}[k, l], \beta=\beta_{d}[k, l]} \\
& \text { PostSUTSamp }[k, l]=\tau_{\text {System }}\left(\alpha_{d}[k, l], \beta_{d}[k, l]\right) \times \\
& \left(\sum_{i=0}^{N_{i}-1 N_{j}-1} \operatorname{PostNU}[i, j] \operatorname{System}\left(\alpha_{d}[k, l]-\frac{i-\delta i_{\text {Array }}}{i \text { Scale }_{\text {Array }}}, \beta_{d}[k, l]-\frac{j-\delta j_{\text {Array }}}{j \text { Scale }_{\text {Array }}}\right)\right)
\end{aligned}
$$

\subsection{Restriction of Summation Limits}

The primary bottleneck in calculating the PostSUTSamp image above is the double summation extending over the entire resistor array for each $[k, l]$ detector. However, for a given $[k, l]$ detector there is only a need to sum up a limited number of $i$ 's (resistor array rows) and $j$ 's (resistor array columns) based on the fact that the rolledup System function will be effectively zero outside the range $\left(\alpha_{\min }<\alpha<\alpha_{\max }\right.$, $\left.\beta_{\text {min }}<\beta<\beta_{\max }\right)$.

$$
\begin{aligned}
& \text { PostSUTSamp }[k, l]=\tau_{\text {System }}\left(\alpha_{d}[k, l], \beta_{d}[k, l]\right) \times \\
& \left(\sum_{i=i_{\min }}^{\left.i_{\max }[k, l] l\right]} \sum_{j=j_{\min }[k, l]}^{j_{\max }[k, l]} \operatorname{PostNU}[i, j] \operatorname{System}\left(\alpha_{d}[k, l]-\frac{i-\delta i_{\text {Array }}}{i \text { Scale }_{\text {Array }}}, \beta_{d}[k, l]-\frac{j-\delta_{j_{\text {Array }}}}{j \text { Scale }_{\text {Array }}}\right)\right)
\end{aligned}
$$

In the above form, the output as discretely sampled by the SUT can be calculated and viewed as desired.

\subsection{Complete IRSP System Model}

The final modeled representation of the IRSP takes the form: 


$$
\text { Output }[k, l]=\operatorname{SUTDig}\{\text { SUTNoise }\{\text { PostSUTSamp }[k, l]\}\}
$$

where:

PostSUTSamp $[k, l]=\tau_{\text {Sumer }}\left(\alpha_{\lrcorner}[k, l], \beta_{d}[k, l]\right) \times$

$$
\left(\sum_{\left.i=\sum_{\min } k, l\right]}^{i_{\max }[k, l]} \sum_{j=\min _{\min }[k, l]}^{j_{\max }[k, l]} \operatorname{PostNU}[i, j] \operatorname{System}\left(\alpha_{d}[k, l]-\frac{i-\delta i_{\text {Array }}}{i \text { Scale }_{\text {Aray }}}, \beta_{d}[k, l]-\frac{j-\delta j_{\text {Array }}}{j \text { Scale }_{\text {Aray }}}\right)\right)
$$

$\operatorname{PostNU}[i, j]=\operatorname{ArrayNU}\{$ SubHeat $\{$ BusBarRob $\{\operatorname{AIE}[]\}\}, N U \operatorname{Coefs}[i, j, 1 \ldots N]\}$

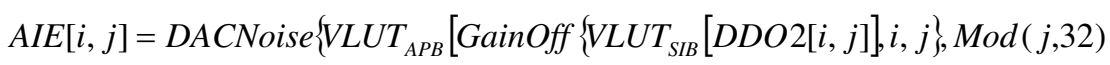

\section{Conclusion and Closing Remarks}

In it's current state, the IDM incorporates the IRSP effects that tend to redistribute the image energy as detected by the SUT. These types of effects are: resistor fill factor, optical distortion, optical geometric aberrations, SUT diffraction, and SUT geometric aberrations. Over the next couple of years the IDM will grow to encompass the rest of the IRSP effects.

Using the equations described above requires detailed knowledge of the IRSP configuration and components. Example results of using the IDM based on a real IRSP can be found in the simultaneously published paper: "Infrared Scene Projector Digital Model Development". Unfortunately, publication limitations have precluded the derivations of the scale factors, limits, and other parameters used in the IDM. Interested parties are encouraged to contact the primary author for additional information. 